\title{
A semiquantitative color Doppler ultrasound scoring system for evaluation of synovitis in joints of patients with blood-induced arthropathy
}

Ningning Zhang ${ }^{1}$, Sheng Yang ${ }^{2}$, Anne-Fleur Zwagemaker ${ }^{3}$, Aihua Huo ${ }^{1}$, Ying-Jia Li ${ }^{4}$, Fang Zhou ${ }^{4}$, Pamela Hilliard ${ }^{5}$, Sandra Squire ${ }^{6}$, Vanessa Bouskill ${ }^{7,8}$, Arun Mohanta ${ }^{9}$, Alex Zhou ${ }^{9}$, Jose Jarrin ${ }^{9}$, Runhui Wu ${ }^{10}$, Jing Sun ${ }^{4}$, Brian Luke ${ }^{11}$, Rahim Moineddin ${ }^{12}$, Victor S. Blanchette ${ }^{7,8}$, Yun Peng ${ }^{1 *+}$ and Andrea S. Doria ${ }^{9,13^{*+}}$ (D)

\begin{abstract}
Background: Intra-articular bleeds in patients with inherited bleeding disorders lead to active synovitis which may progress to a chronic state over time. We explored the diagnostic value of color Doppler ultrasound in detecting synovitis in boys with bleeding disorders.

Results: Sixty boys with hemophilia and 3 boys with type 3 von Willebrand disease aged 5 to 18 years (median 12.3 years) were imaged by gray-scale and color Doppler ultrasound (US) in three centers (Beijing, China [ $n=22]$, Guangzhou, China $[n=12]$ and Toronto, Canada $[n=29])$ ) in this observational study. Images were independently reviewed by two radiologists blinded to clinical data using a subjective semi-quantitative scoring system and objective measurements of synovial thickness and vascularity. Inter-reader reliability for using subjective versus objective color Doppler US methods for assessing synovial vascularity was excellent for the subjective method and moderate/ lower range of substantial for the objective method. Agreement between degree of vascularity on color Doppler and extent of synovial hypertrophy on gray-scale US was overall poor for Canada data and moderate for China data. Correlations between degree of vascularity on color Doppler and synovial hypertrophy on gray-scale US, and clinical constructs (total and itemized HJHS scores and total Pettersson X-ray scores) for assessment of blood-induced arthropathy were all poor.
\end{abstract}

Conclusion: Color Doppler US is a valuable scoring method for evaluating reactive synovitis in joints of subjects with inherited bleeding disorders and holds potential for assessing post-bleed reactive synovitis once further information on its association with timing of the joint bleed becomes available in the literature.

Keywords: Color Doppler ultrasound, Synovial hypertrophy, Magnetic resonance imaging (MRI), Hemophilic arthropathy, Children and adolescents.

\footnotetext{
*Correspondence: ppengyun@hotmail.com; andrea.doria@sickkids.ca †Yun Peng and Andrea S. Doria are sharing senior authorship ${ }^{1}$ Department of Radiology, Beijing Children's Hospital, Capital Medical University, National Center for Children's Health, Beijing, China ${ }^{9}$ Department of Diagnostic Imaging, Research Institute, The Hospital for Sick Children, University of Toronto, 555 University Ave, Toronto, ON M5G 1X8, Canada

${ }^{13}$ Department of Medical Imaging, University of Toronto, Toronto, ON, Canada

Full list of author information is available at the end of the article
}

\section{Key points}

- Active synovitis due to intra-articular bleeds may progress to a chronic state.

- Different correlation levels were obtained while comparing subjective versus objective assessing methods. 
- Color Doppler US holds potential for assessing postbleed reactive synovitis.

\section{Background}

Recurrent joint bleeding and subsequent development of joint damage are complications in persons with moderate or severe hemophilia, in whom hemarthroses account for $70-80 \%$ of all bleeding episodes [1]. Intra-articular bleeds lead initially to active synovitis, characterized by hypervascularization and inflammatory activity, and microscopic degenerative changes in the cartilage. Over time, there may be progression to a chronic state of synovitis, where macroscopic cartilage erosions and arthropathy may be observed $[2,3]$. Due to the occurrence of subclinical bleeding, early pathological synovial changes may go unrecognized. Nevertheless, such subtle undiagnosed synovial abnormalities may lead to irreversible arthropathy later on [4]. Hence, accurate monitoring of joint status is an essential part of hemophilia care in order to guide treatment decision-making.

Although contrast-enhanced magnetic resonance imaging (MRI) is a sensitive imaging technique to assess early synovial joint disease in hemophilia [5], its applicability is restricted by high costs for data scanning and interpretation, and the need for sedation in young children, and for administration of contrast for assessment of synovitis. Technologic advances in real-time sonography have facilitated the use of gray-scale sonography and color Doppler for evaluating the musculoskeletal system [6-8]. Ultrasound with color Doppler can be a useful non-invasive technique in this regard. Acharya et al. have shown that power Doppler ultrasound is an inexpensive imaging tool for detecting hemophilic synovitis and for quantifying synovial vascularity in knees, elbows and ankles of persons with hemophilia [9]. Color and power Doppler ultrasound hold potential for detection and quantitation of increased vascularity in joints of persons with hemophilia related to synovitis which can be reactive to recent intraarticular bleeds. This has been previously shown in animal $[10,11]$ and human [12] studies. Power Doppler has improved sensitivity compared with conventional Doppler frequency displays [13], however it shows a high degree of frame-averaging requiring that the patient keeps still during the performance of the examination, a task that can be challenging for young children. The contrast resolution and sensitivity of modern color flow systems have rapidly improved over the last decade, improving the range and quality of vascular examination [14]. Torp-Pedersen et al. assessed 6 different types of high- and intermediate-range ultrasound machines to determine how settings for power and color Doppler ultrasound sensitivity varied in patients with rheumatoid arthritis affecting the wrist joint. Their study results showed that whereas power Doppler was more sensitive on half of the machines color Doppler was more sensitive on the other half, using both manufacturer and local study settings [15]. Because color Doppler is substantially less affected by motion artifacts than power Doppler it is preferentially used in the assessment of pediatric patients in some centers.

Although rheumatoid arthritis and juvenile idiopathic arthritis (JIA) are characterized by prominent synovitis and hemophilic arthritis by minor synovitis, the joint disease in these entities shows similarities in both radiographic and histological appearance [16, 17]. In JIA and adult rheumatoid arthritis, color pixel intensity obtained with color and power Doppler ultrasound was found to be markedly increased in patients with active disease and patients having quiescent disease with serum markers of active disease $[18,19]$. In blood-induced arthritis, animal studies have shown that ongoing or repeat joint bleed induces synovial changes that include inflammation, hyperplasia and angiogenesis [20-22]. Acharya et al. noted local and systemic angiogenic response in hemophilic subjects with recurrent hemarthroses points towards mechanisms of onset and progression of hemophilic synovitis [23]. As neoangiogenesis and inflammatory processes require increased blood flow [23], the presence of synovial hyperemia on color Doppler ultrasound can be a biomarker of active synovitis raising the hypothesis that Doppler sonography may add value in the assessment of arthropathy in persons with hemophilia.

Concerning the use of scoring systems, previous studies have used color Doppler scoring systems for assessment of small joints in the hands and feet of subjects with rheumatoid arthritis [24]. However, to our knowledge, few if any previous studies have evaluated the clinical value of color Doppler ultrasound in comparison to MRI for assessment of reactive synovitis in index joints (ankles, knees and elbows) in subjects with hemophilia using an a priori devised standardized color Doppler scoring system.

The specific aims of this study were (i) to evaluate the agreement and associations between different color Doppler methods for assessment of the maximum degree of synovial vascularity, a semi-quantitative scoring method (subjective assessment) and quantitative pixel measurements (objective assessment), and (ii) to correlate the degree of vascularity on color Doppler (by semi-quantitative and quantitative methods), and the extent of synovial hypertrophy on gray-scale ultrasound, with clinical/ other imaging constructs (Hemophilia Joint Health Score [HJHS], Pettersson X-ray scores and MRI of the study joint). 


\section{Methods}

Research Ethics Board approval for the study was obtained in all participating centers. The approval dates were respectively April 2, 2007 (for the prospective part) and February 5, 2015 (for the current study including research agreements with the centers China) in Toronto, Canada; December 29, 2014 in Beijing, China and December 25, 2014 in Guangzhou, China. Informed consent for participation in the study was obtained for all subjects.

\section{Patients}

Eligibility for this study included patients with bleeding disorders (hemophilia A, hemophilia B and von Willebrand [vWD] disease) with ages ranging between 0 and 18 years. The ultrasound examinations whose data were used in this study were prospectively acquired in Canada and in China. The recruitment period of patients in Toronto, Canada was September 19, 2007 to January 23, 2009; in Beijing, China, February 7 to 12, 2015 and in Guangzhou, China, February 11 to 13, 2015. Afterwards, the data were retrospectively accrued for the purpose of this study, based on an opportunity sample size. This has limited the assessment of timing of intraarticular bleeds through a rigorous methodology that enabled uniform documentation in patients' clinical records.

\section{Imaging acquisition}

Ultrasound, MRI and physical examination of the study joints were obtained on the same day, and radiographs of the corresponding study joint were obtained within an interval of 3 days of the other diagnostic tests. Images were acquired in Toronto during the period of September 2007 to January 2009 and in China in February 2015.

\section{Ultrasound}

Gray and color Doppler ultrasound images were obtained of ankles, knees and elbows according to protocols previously described [25-27]. The protocol included sagittal, coronal and axial images obtained with depths and gains adjustable to the patients' biotypes. Sites 1 Beijing Children's Hospital, Beijing and 2, Nanfang Hospital, Guangzhou, in China used a 12-5-MHz linear-array transducer on an iU22 scanner (Philips Medical Systems, Bothell, WA), and site 3, Hospital for Sick Children, Toronto, Canada used a 17-5 MHz linear-array transducer on an iU22 scanner (Philips Medical Systems, Bothell, WA). Each ultrasound scan was performed by two operators blinded to any clinical or additional imaging information: in the Chinese hemophilia treatment centers (Beijing Children's Hospital, Beijing and Nanfang Hospital, Guangzhou) where the data were acquired between February
7 and 13, 2015 one operator was from China (in site 1, N.Z. and in site 2, Y-J.L) and one from Canada (in site 1, A.M. and in site 2, J.J. or A.Z.), and in the Canadian hemophilia treatment center (Hospital for Sick Children, Toronto) where the data were acquired between September 19, 2007 and January 23, 2009, the two operators were from Canada (A.M. and J.J.).

The most severely affected joint (knee, elbow or ankle) of the participating patients as per clinical history and prior X-rays, MRI, and ultrasound scans, according to a priori established protocols, were scanned in this study [25-27]. Scanning time was approximately $20 \mathrm{~min}$ for ankles and elbows, and $30 \mathrm{~min}$ for knees.

\section{MRI}

MRI assessments were obtained on 3.0 Tesla scanners: in site 1 investigators used an Achieva TX (Philips Medical Systems, Bothell, WA) with an 8-channel coil for knees, a flexible coil for elbows and a head coil for ankles. In sites 2 and 3 a Trio Tim system (Siemens, Erlangen, Germany) was employed with a 15-channel coil for knees, a flexible coil for elbows and a head coil for ankles. MRI scanning protocols used in the three hemophilia treatment centers are detailed in the Additional file 1: Table 1.

\section{Imaging analysis \\ Gray scale ultrasound}

Quantitative assessment Synovial thickness was measured on the plane and image that depicted the area with the most severely hypertrophic synovium by a single operator (S.Y.).

\section{Color Doppler ultrasound}

Synovial vascularity of study joints was measured on the plane that depicted the most marked vascularity both quantitatively and semi-quantitatively by two operators (N.Z., S.Y.).

Quantitative assessment Geometric regions-of-interest (ROIs) encompassing unique color pixel areas for each case were manually drawn within the color boxes to quantify the pixel intensity on color Doppler, including area of pixels (in $\mathrm{mm}^{2}$ ) and the pixel count as provided by the built-in software of our Picture Archive Communications Systems (PACS), General Electric, Milwaukee, WI, U.S.A.

Semi-quantitative assessment The degree of vascularity of a given joint on color Doppler ultrasound was scored based on the image that showed most severe findings regardless of location in the joint and included the number of color pixels and extent of vascularity seen within the ROI (Figs. 1, 2) according to a semi-quantitative score, modified from Backhaus et al. [24] as fol- 

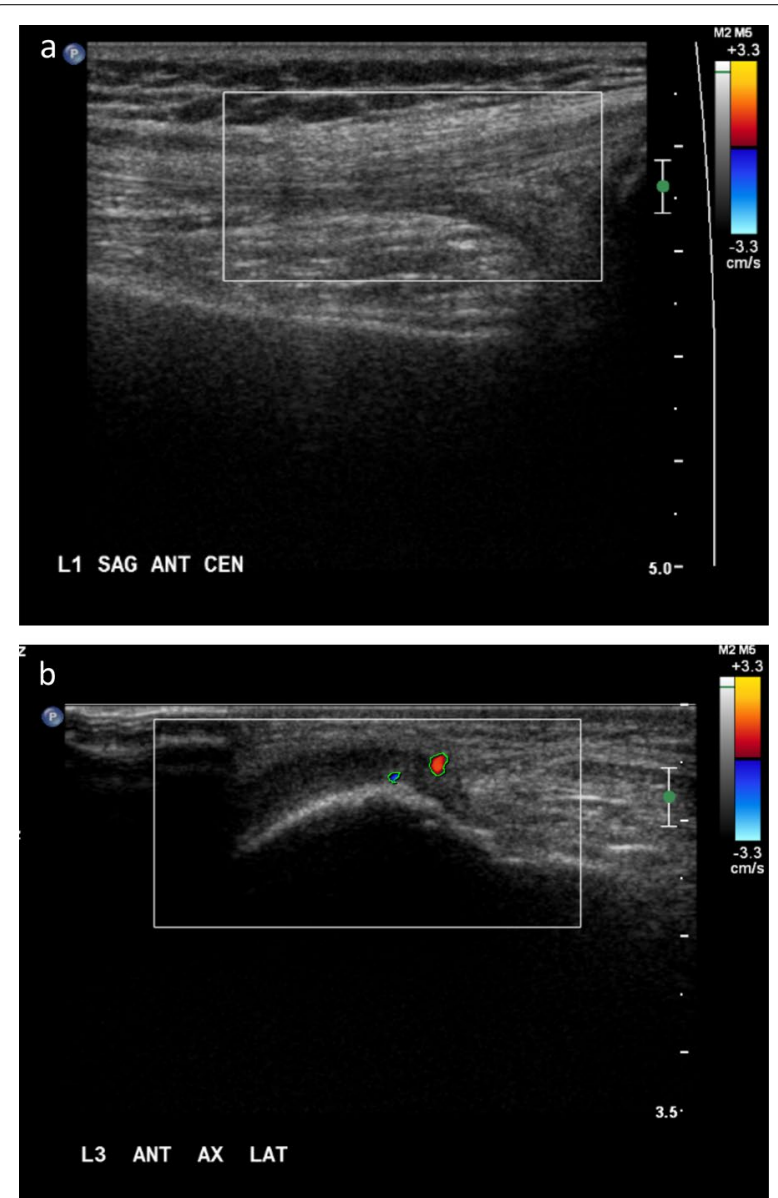

Fig. 1 Normal synovial vascularity of joints of patients with

hemophilic arthropathy on color Doppler ultrasound. a Thirteen-year old boy with severe hemophilia A and a history of recurrent bleeding into his right knee. Color Doppler sonogram obtained on the sagittal anterior central plane at the L1 level shows no color pixel dot within the color box. Score is 0 . Objective area of pixels is 0 , score equals 0 . b Fourteen-year old boy with severe hemophilia A and a history of recurrent bleeding into his right knee. Color Doppler sonogram obtained on the axial anterior-lateral plane at the L3 level shows

2 color pixel dots (less than 4 dots) within the color box, Score equals 0 . The area of color pixels within the color box is 569 units $^{2}$

lows: 'normal' (grade 0), 'mild to moderate' (grade 1) and 'severe' (grade 2) (Table 1). Two radiologists with 8 and 6 years of experience in ultrasound after training, respectively (reviewer 1, N.Z.; reviewer 2 , S.Y.), evaluated each assigned image according to the a priori determined criteria (Table 1). They both completed a tutorial session for calibration of measurements on color Doppler images of joints before formal reading of the study images. Discrepancies in results were resolved by an adjudicator (A.S.D.), a radiologist with over 10 years of experience after training.
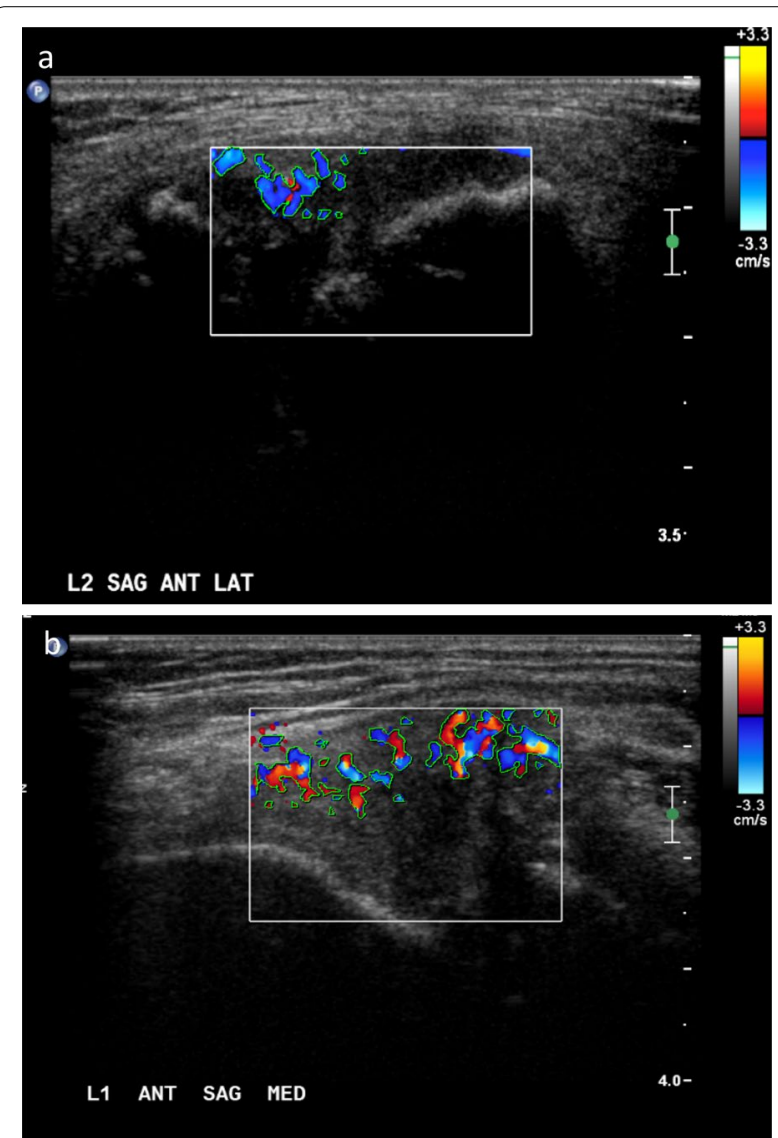

Fig. 2 Increased synovial vascularity of joints of patients with hemophilic arthropathy on color Doppler ultrasound. a Eight-year old boy with severe hemophilia A and a history of prior bleeding into his right ankle. Mild hypervascularity is seen in his joint on color Doppler ultrasound. Color Doppler sonogram obtained on the sagittal anterior-lateral plane at the L2 level shows more than 4 color pixel dots encompassing less than $50 \%$ of the synovium extent within the color Doppler box which represents some degree of synovitis. Score equals 1. The area of color pixels within the color box is 4,257 units $^{2}$. b Twelve-year old boy with severe hemophilia A and a history of recurrent bleeding into his left elbow. Extensive hypervascularity is seen in his joint on color Doppler ultrasound. Color Doppler sonogram obtained on the sagittal anterior medial plane at the L1 level shows more than 4 color pixel dots encompassing 50\% or more of the synovium extent within the color Doppler box. Score equals 2. The area of color pixels within the color box is 13,605 units ${ }^{2}$

\section{MRI}

Like measurements attained on gray-scale ultrasound images, synovial thickness was measured on the MRI plane and image that depicted the area with most severe hypertrophic synovium by a single operator (A.F.Z.).

\section{Physical examination}

The most affected joint underwent physical examination according to the Hemophilia Joint Health Score (HJHS) version 2.1 by an experienced score-trained 
Table 1 Color Doppler semi-quantitative scoring for assessment of ankles, elbows and knees of patients with blood-induced arthropathies

\begin{tabular}{lll}
\hline Grades & Criteria & Score \\
\hline Normal (grade 0) & Normal: $<4$ dots within box & 0 \\
Mild to moderate (grade 1) & $\geq 4$ dots within box and $<50 \%$ of ROI filled with color pixels representing hyperemia & 1 \\
Severe (grade 2) & $\geq 50 \%$ of ROl filled with color pixels representing hyperemia & 2 \\
\hline
\end{tabular}

Criteria: Based on the number of dots (color pixels) and extent of vascularity at the location with most severe findings and extent of vascularity within the color Doppler box

ROI, region-of-interest

physiotherapist in each of the two sites in China [28] (Additional file 1: Table 2). The HJHS version 2.0 was used for the site in Canada and scores were converted into the HJHS version 2.1 scale by the study physiotherapist in Toronto.

\section{Statistical analysis}

Count and area of pixels on color Doppler were reported as mean, median and range/standard deviation (SD) as appropriate.

The inter-reader reliability for the utilization of either a semi-quantitative scoring method (subjective assessment by experienced readers) or a quantitative method of measurement of color pixels (objective assessment) reflecting the maximum degree of vascularity on color Doppler was analyzed by intraclass correlation coefficients (ICCs) for continuous data, Kendall's coefficient of concordance for ordinal data and kappa coefficients for nominal data $(<0.40$, poor agreement; $\geq 0.40$ and $<0.60$, moderate agreement; $\geq 0.60$ and $<0.80$, substantial agreement; and $\geq 0.80$, excellent agreement) [29]. Associations between the color Doppler synovial scores (subjective assessment), quantitative color Doppler pixel measurements (objective assessment) and quantitative grayscale US measurements of synovial hypertrophy were compared among themselves and with total Hemophilia Joint Health Score (HJHS) scores: presence/absence of swelling, duration of swelling, flexion/extension loss and pain item of the HJHS score. Pettersson X-ray scores were calculated with Spearman correlation coefficients ( $r$ values) for continuous data. $r$ values $<0.40$ indicated poor, $\geq 0.40$ and $<0.60$ moderate, $\geq 0.60$ and $<0.80$ substantial and $\geq 0.80$ excellent agreement [29]. For assessment of associations between continuous and categorical variables analysis of variance (ANOVA) was utilized.

In some joints in the China and Canada datasets, the synovium thickness could not be reliably assessed by the operators either due to technical limitations or to extremely reduced thickness and represented missing data. Correlations for synovial thickness measured on US in this study took into consideration available values in each dataset. Concerning demographic information of study subjects information on missing data for baseline factor VIII/IX and HJHS scores is available in Additional file 1: Table 2.

$p$ values $<0.05$ were considered statistically significant. We used SAS ${ }^{\circledR}$ system software package, version 9.4 (SAS Institute Inc. Cary, NC) for all analyses.

\section{Results}

Table 2 and Additional file 1: Table 2 shows demographic and clinical characteristics of study subjects. A total of 63 children and adolescents with ages ranging from 5 to 18 years, median age 12.3 years agreed to participate in the original studies conducted at the three participating hemophilia treatment centers and were imaged by gray and color Doppler ultrasound, 34 in China (site 1, Beijing [ $n=22]$; site 2, Guangzhou $[n=12]$; and 29 in Canada (site 3, Toronto). Sixty cases of our cohort were boys with severe or moderate hemophilia A $[n=52]$ or $\mathrm{B}[n=8]$. Forty one cases had factor VIII/IX levels of $<1 \%$ and 19 cases had factor VIII/IX levels of $1-5 \%$. Three patients had von Willebrand disease (VWD) type 3, a severe form of VWD. There was a good balance of ankles (35.2\%, 12 out of 34$)$, knees (32.4\%, 11 out of 34$)$ and elbows $(32.4 \%, 11$ out of 34$)$ studied in the Chinese cohort but for the Canadian cases the majority of joints studied were ankles $(71.4 \%$, 20 out of 28 ).

As expected the severity of joint disease at the time of the imaging studies was discordant between the Canadian and Chinese cases: 61.7\% (21 out of 34) of the Chinese cases had joints with total HJHS scores of 4 or higher versus $37.9 \%$ (11 out of 29 ) of cases from Canada. This almost certainly reflects the fact most boys $<18$ years of age in Canada with moderate/severe hemophilia would have been on some form of longterm prophylaxis for several years whereas few boys with moderate/severe hemophilia in China studied in 2015 would have been on long-term prophylaxis for a significant period of time.

The inter-reader reliability for using different color Doppler methods for assessment of the maximum degree of synovial vascularity, a semi-quantitative 
Table 2 Demographic and clinical characteristics of study subjects

\begin{tabular}{|c|c|c|c|c|c|c|c|c|c|}
\hline Study site & $N$ & $\begin{array}{l}\text { Age (range, } \\
\text { median) }\end{array}$ & $\begin{array}{l}\text { Ankles } \\
\text { Imaged }\end{array}$ & $\begin{array}{l}\text { Elbows } \\
\text { imaged }\end{array}$ & $\begin{array}{l}\text { Knees } \\
\text { imaged }\end{array}$ & $\begin{array}{l}\text { Hemophilia } \\
\text { type }\end{array}$ & $\begin{array}{l}\text { Baseline } \\
\text { FVIII/IX } \\
\text { Level* (\%) }\end{array}$ & $\begin{array}{l}\text { Pettersson } \\
\text { score }\end{array}$ & $\begin{array}{l}\text { HJHS } \\
\text { score (range, } \\
\text { median) }\end{array}$ \\
\hline \multirow[t]{5}{*}{1} & 22 & $7-17,13$ & 8 & 7 & 7 & $A: N=17$ & $0: N=13$ & Score $0: N=1$ & $7-15,9$ \\
\hline & & & & & & & $1: N=4$ & Scores $1-3: N=8$ & \\
\hline & & & & & & & & Scores 4-7:N=5 & \\
\hline & & & & & & $B: N=5$ & $0: N=4$ & Scores $\geq 8: N=8$ & \\
\hline & & & & & & & $1: N=1$ & & \\
\hline \multirow[t]{4}{*}{2} & 12 & $8-18,11.5$ & 4 & 4 & 4 & $A: N=12$ & $0: N=5$ & Score $0: N=0$ & $3-16,11.5$ \\
\hline & & & & & & & $1: N=7$ & Scores $1-3: N=4$ & \\
\hline & & & & & & & & Scores $4-7: N=4$ & \\
\hline & & & & & & & & Scores $\geq 8: N=4$ & \\
\hline \multirow[t]{5}{*}{3} & 29 & $5-17,13$ & 21 & 0 & 8 & $A: N=23$ & $0: N=20$ & Score $0: N=14$ & $0-16,4.5$ \\
\hline & & & & & & & $1: N=3$ & Scores 1-3:N=4 & \\
\hline & & & & & & $B: N=3$ & $0: N=2$ & Scores $4-7: N=5$ & \\
\hline & & & & & & & $1: N=1$ & Scores $\geq 8: N=6$ & \\
\hline & & & & & & $\begin{array}{l}\text { vWD type } \\
3: N=3^{* *}\end{array}$ & & & \\
\hline
\end{tabular}

$\mathrm{N}$, number; FVIII/IX, factors VIII/IX; IU, International Unit; dL, deciliter; HJHS, Hemophilia Joint Health Score

*Baseline FVIII/IX level: $0:<1 \mathrm{IU} / \mathrm{dL}$ (=<1\%, consistent with a diagnosis of severe hemophilia) 1: 1-5 IU/dL (=1-5\%, consistent with a diagnosis of moderate hemophilia)

**Three patients had vWD

Table 3 Inter-reader reliability for using different color Doppler methods (subjective, semi-quantitative and objective, quantitative) for assessment of maximal synovial vascularity in joints of patients with blood-induced disorders

\begin{tabular}{lll}
\hline Testing domain & US operators (data acquisition) & $\begin{array}{c}\text { Reliability [ICC (95\% Cls)] for } \\
\text { interpretation of data by readers } \\
(\mathbf{1}, \mathbf{2})\end{array}$ \\
\hline Semi-quantitative scoring system & Canada & $1.00(1.00,1.00)$ \\
& China & $0.98(0.97,0.98)$ \\
Quantitative scoring system & China & $0.61(-0.51,0.96)$ \\
& Area of pixels & $0.60(-0.52,0.96)$ \\
& Count of pixels & $0.67(0.43-0.82)$ \\
& Canada & $0.70(0.48-0.84)$
\end{tabular}

US, ultrasound; ICC, intraclass correlation coefficients; $\mathrm{Cl}$, confidence intervals

scoring method (subjective assessment) and quantitative pixel measurements (objective assessment) was excellent for the semi-quantitative method and moderate/lower range of substantial for the quantitative method regardless of the origin of the data acquisition (Table 3).

Concerning the association between the two aforementioned color Doppler analytic methods, semiquantitative and quantitative scoring systems, results depended most on the reader who defined the ROIs than on the origin of the data. Whereas highly substantial correlations were noted for both Canada and China data between the two methods when reader 1 defined the ROI, moderate to substantial correlations were noted for Canada and China data, respectively, when ROIs were defined by reader 2 (Table 4).

Table 5 shows mean thickness of single layer synovium on gray-scale US and mean count of pixels in the 
Table 4 Associations between a subjective semi-quantitative color Doppler US scoring method (A), a quantitative color Doppler method for synovial vascularity (B) and a quantitative gray-scale US method for single-layer synovial hypertrophy (C)

\begin{tabular}{|c|c|c|c|c|}
\hline \multirow[t]{2}{*}{ Origin of patient's data } & $\begin{array}{l}\text { Correlation coefficient } r(95 \% \\
\text { Cls) }\end{array}$ & $p$ value & $\begin{array}{l}\text { Correlation coefficient } r(95 \% \\
\text { Cls) }\end{array}$ & $p$ value \\
\hline & \multicolumn{2}{|l|}{ A vs $B$ methods } & \multicolumn{2}{|l|}{ B vs C methods } \\
\hline \multicolumn{5}{|l|}{ A. Canada US operator } \\
\hline & Reader 1 & & Reader 1 & \\
\hline Canada Data & $0.72(0.47,0.86)$ & $<0.0001$ & $0.43(0.08,0.69)$ & 0.02 \\
\hline \multirow[t]{2}{*}{ China Data } & $0.76(0.56,0.87)$ & $<0.0001$ & $0.66(0.40,0.82)$ & $<0.0001$ \\
\hline & Reader 2 & & Reader 2 & \\
\hline Canada Data & $0.63(0.34,0.81)$ & 0.0001 & $0.44(0.08,0.69)$ & 0.02 \\
\hline China Data & $0.79(0.62,0.89)$ & $<0.0001$ & $0.60(0.34,0.78)$ & $<0.0001$ \\
\hline \multicolumn{5}{|l|}{ B. China US operator } \\
\hline & Reader 1 & & Reader 1 & \\
\hline Canada Data & $0.63(0.34,0.81)$ & 0.0001 & $0.44(0.08,0.69)$ & 0.0151 \\
\hline \multirow[t]{2}{*}{ China Data } & $0.79(0.62,0.89)$ & $<0.0001$ & $0.60(0.34,0.78)$ & $<0.0001$ \\
\hline & Reader 2 & & Reader 2 & \\
\hline Canada Data & $0.84(0.68,0.92)$ & $<0.0001$ & $0.64(0.35,0.81)$ & $<0.0001$ \\
\hline China Data & $0.85(0.71,0.92)$ & $<0.0001$ & $0.61(0.33,0.79)$ & 0.0001 \\
\hline
\end{tabular}

Bold indicates statistically significant results. $p$ values $<0.05$ were considered statistically significant

US, ultrasound; $\mathrm{Cl}$, confidence intervals

Table 5 Mean thickness of synovium and mean count of color Doppler pixels in the perisynovial regions of joints of patients from Canada and China centers according to origin of ultrasound operators and patients' data

\begin{tabular}{llllcc}
\hline $\begin{array}{l}\text { Origin of } \\
\text { ultrasound } \\
\text { operators }\end{array}$ & $\begin{array}{l}\text { Origin of } \\
\text { patients' } \\
\text { data }\end{array}$ & $\begin{array}{l}\text { Mean thickness of } \\
\text { synovium }(\mathbf{m m})-\text { gray- } \\
\text { scale US }\end{array}$ & $\begin{array}{l}\boldsymbol{p} \text { value (between values } \\
\text { according to origin of patients' } \\
\text { data) }\end{array}$ & $\begin{array}{l}\text { Mean count of } \\
\text { color Doppler } \\
\text { pixels }\end{array}$ & $\begin{array}{l}\boldsymbol{p} \text { value (between values } \\
\text { according to origin of patients' } \\
\text { data) }\end{array}$ \\
\hline Canada & Canada & 2.1 & $P<0.0001$ & 930 & $P=0.003$ \\
Canada & China & 4.1 & $P<0.0001$ & 3,633 & $P=0.002$ \\
China & Canada & 2.0 & & 868 & 3,796 \\
China & China & 3.9 & & \\
\hline
\end{tabular}

Single layer synovium was considered in the data analysis

Color Doppler pixel count was used as an outcome of synovial vascularity

US, ultrasound. $p$ values $<0.05$ were considered statistically significant

peri-synovial region of joints of patients on color Doppler US. Differences were noted for mean synovial thickness when data from patients of Canada and China were compared, regardless of the country of origin of US operators ( $p<0.0001$ for each comparison) and also for mean count of color Doppler pixels between data from China and Canada patients, regardless of the country of origin of US operators $(p=0.003$ and $p=0.002$, for Canada and China US operators, respectively).

Concerning the association between the quantitative degree of vascularity on color Doppler and the extent of synovial hypertrophy on gray-scale ultrasound correlations were consistently at lower limits of moderate for Canada data and upper limits of moderate to substantial for China data for Canadian US operators slightly improving for Canada data when Chinese US operators obtained the data. These correlations were maintained regardless of the reader who performed measurements (Table 4).

Correlations for synovial thickness measured on US between Canadian and Chinese readers were excellent for China data $(r=0.93, p<0.0001)$ and substantial for Canada data $(r=0.77, p=0.0001)$. Nevertheless, correlations for synovial thickness measured on MRI and US were moderate for China data $(r=0.64, p=0.001$, Canadian US operator and $r=0.63, p=0.001$, Chinese US operator) and not significant for Canada data $(r=0.05$, $p=0.86$, Canadian US operator and $r=0.46, p=0.10$, China US operator). Note is made of the fact that in 10 out of 34 (29.4\%) available cases of the China dataset and 
Table 6 Associations between the degree of vascularity on color Doppler and clinical /imaging constructs Hemophilia Joint Health Score (HJHS) of the study joint, Pettersson score of the study joint

\begin{tabular}{|c|c|c|c|c|c|c|c|}
\hline \multirow{2}{*}{$\begin{array}{l}\text { US operators } \\
\text { (data } \\
\text { acquisition) }\end{array}$} & & \multirow{2}{*}{$\begin{array}{l}\text { Correlation coefficient } \\
r(95 \% \text { Cls) } \\
\text { Semi-quantitative } \\
\text { color Doppler US } \\
\text { scoring method } \\
\text { (synovial vascularity) }\end{array}$} & \multirow[t]{2}{*}{$p$ value } & \multirow{2}{*}{$\begin{array}{l}\text { Correlation coefficient } \\
r(95 \% \text { CIs) } \\
\text { Quantitative color } \\
\text { Doppler US pixel } \\
\text { measurement } \\
\text { (synovial vascularity) }\end{array}$} & \multirow[t]{2}{*}{$p$ value } & \multirow{2}{*}{$\begin{array}{l}\text { Correlation coefficient } \\
r(95 \% \text { Cls) } \\
\text { Quantitative } \\
\text { gray-scale US } \\
\text { measurement } \\
\text { (single-layer synovial } \\
\text { hypertrophy) }\end{array}$} & \multirow[t]{2}{*}{$p$ value } \\
\hline & & & & & & & \\
\hline Canada & $\begin{array}{l}\text { Total Pettersson X-rays } \\
\text { scores }\end{array}$ & $0.14(-0.25,0.48)$ & 0.49 & $0.04(-0.34,0.40)$ & 0.85 & $0.24(-0.15,0.56)$ & 0.22 \\
\hline China & $\begin{array}{l}\text { Total Pettersson X-rays } \\
\text { scores }\end{array}$ & $0.29(-0.06,0.57)$ & 0.09 & $0.11(-0.25,0.44)$ & 0.55 & $0.25(-0.10,0.54)$ & 0.15 \\
\hline Canada & Total HJHS scores & $0.21(-0.20,0.55)$ & 0.30 & $0.31(-0.09,0.62)$ & 0.12 & $0.53(0.16-0.75)$ & 0.005 \\
\hline China & Total HJHS scores & $0.32(-0.10,0.63)$ & 0.12 & $-0.01 .(-0.41,0.42)$ & 0.97 & $0.16(-0.26-0.52)$ & 0.46 \\
\hline Canada & $\begin{array}{l}\text { Presence/absence of } \\
\text { swelling (item of HJHS } \\
\text { score) }\end{array}$ & $0.32(-0.06,0.61)$ & 0.09 & $0.38(0.01,0.65)$ & 0.04 & $0.27(-0.12-0.57)$ & 0.16 \\
\hline China & $\begin{array}{l}\text { Presence/absence of } \\
\text { swelling }\end{array}$ & $-0.16(-0.50,0.23)$ & 0.41 & $-0.20(-0.54,0.21)$ & 0.34 & $-0.05(-0.41-0.33)$ & 0.81 \\
\hline Canada & $\begin{array}{l}\text { Duration of swelling } \\
\text { (item of HJHS score) }\end{array}$ & $0.10(-0.28,0.45)$ & 0.61 & $0.23(-0.16,0.54)$ & 0.24 & $0.06(-0.32-0.41)$ & 0.77 \\
\hline China & Duration of swelling & $-0.01(-0.39,0.36)$ & 0.94 & $0.12(-0.28,0.48)$ & 0.56 & $-0.06(-0.42-0.32)$ & 0.77 \\
\hline Canada & $\begin{array}{l}\text { Flexion loss (item of } \\
\text { HJHS score) }\end{array}$ & $0.24(-0.15,0.55)$ & 0.22 & $0.16(-0.22,0.50)$ & 0.41 & $0.07(-0.31-0.42)$ & 0.72 \\
\hline China & Flexion loss & $0.38(-0.00,0.65)$ & 0.048 & $0.40(0.00,0.68)$ & 0.04 & $0.27(-0.12-0.58)$ & 0.17 \\
\hline Canada & $\begin{array}{l}\text { Extension loss (item of } \\
\text { HJHS score) }\end{array}$ & $0.34(-0.04,0.62)$ & 0.07 & $0.25(-0.13,0.56)$ & 0.18 & $0.39(0.01-0.65)$ & 0.04 \\
\hline China & Extension loss & $0.09(-0.30,0.45)$ & 0.65 & $-0.16(-0.51,0.24)$ & 0.43 & $0.10(-0.29-0.45)$ & 0.61 \\
\hline Canada & $\begin{array}{l}\text { Pain (item of HJHS } \\
\text { score) }\end{array}$ & $0.23(-0.16,0.54)$ & 0.24 & $0.13(-0.25,0.47)$ & 0.52 & $0.34(-0.04-0.62)$ & 0.07 \\
\hline China & Pain & $0.21(-0.18,0.54)$ & 0.28 & $0.09(-0.31,0.46)$ & 0.68 & $0.29(-0.10-059)$ & 0.14 \\
\hline
\end{tabular}

Bold indicates statistically significant results. $p$ values $<0.05$ were considered statistically significant

$\mathrm{Cl}$, confidence intervals; $\mathrm{R}$, correlation coefficient

in 14 out of $29(48.3 \%)$ cases of the Canada dataset synovium thickness could not be reliably measured by MRI representing missing data.

Table 6 shows associations between the degree of vascularity on color Doppler and other total HJHS scores, presence/absence of swelling, duration of swelling, flexion loss, extension loss, and pain (items of HJHS score)] and Pettersson X-ray scores in the assessment of hemophilic arthropathy.

\section{Discussion}

The synovial membrane is richly supplied with blood, vessels, lymphatics and nerves. Ultrasonography is non-invasive, inexpensive and readily available with no reported complications. It is ideal for determining the presence, extent and nature of soft tissue changes in joints of persons with hemophilia. It has become a mainstay diagnostic tool in the evaluation of inflamed joints, including reactive synovitis in subjects with inflammatory arthropathy.

In this study, the synovium thickness, as well as the area and count of pixels were objectively measured by gray scale and color Doppler US, respectively. Moreover, synovitis was subjectively graded by a color Doppler US scoring system. Our results showed excellent agreement for inter-reader reliability between subjective and objective assessments of ROIs of joints. Nevertheless, we should note that participation in a tutorial session for calibration of ROIs of synovial inflammation prior to commencement of measurements is key given the subjectivity for selection of ROIs. Previous imaging studies have shown that the utilization of a tutorial session and atlas improves the scoring of inflammatory findings of joints, particularly for non-experienced operators [30]. Note is made that reader 1 had a previous 6-month dedicated US training in the assessment of hemophilic arthropathy. 
Our study results indicate an overall poor association between the degree of vascularity on color Doppler and the extent of synovial hypertrophy on gray-scale ultrasound for Canada data and moderate agreement for China data (Table 4). One explanation for this is the fact that for China data categories of absent, mild and severe synovial hypertrophy are available in our dataset whereas for Canada data only absent and mild synovial hypertrophy categories are available. High association means that severe synovial hypertrophy is frequently associated with marked synovial hyperemia, mild synovial hypertrophy with a lower degree of hyperemia and absent synovial hypertrophy with absence of synovial color pixels. Although in both datasets absent synovial hypertrophy is associated with 0 or with a small number of color pixels, the lower number of categories of synovial hypertrophy for the Canada dataset and the more frequent presence of color pixels in cases of absent synovial hypertrophy may justify the poorer associations for Canada data. Further normative data on synovial vascularity according to different levels of physical activity of children is urgently needed for us to better understand the definitions of active and non-active synovium and the relationship between degree of synovial hypertrophy and of reactive synovial hypervascularity. The lower HJHS scores of the study joint for Canada data in relation to China data (Table 2) may infer that joints of Chinese patients had more active synovitis due to more severe joint bleeding than joints of Canadian patients which could account for overall less synovial inflammation and lesser grade of synovial hypertrophy seen on Canada data.

Previous studies on the assessment of synovial abnormalities with ultrasound and MRI showed a good agreement between the two techniques [27]. In our study, the inter-reader reliability for measuring maximum degree of synovial vascularity, showed excellent reliability for semi-quantitative assessment, both in Canada and China data, and moderate/lower range of substantial for quantitative assessment in the data from all three hemophilia treatment centers. This suggests that the proposed semiquantitative color Doppler holds more promise for use in clinical practice, regardless of the severity of synovitis. Furthermore, in this study unenhanced MRI examinations were not useful as comparators for synovium thickness in relation to gray-scale US given the impossibility of reliable measurements to be obtained without contrast enhancement and the presence of susceptibility artifacts from hemosiderin deposition on gradient-recalled echo images which obscured fine detail of the synovium. These limitations of MRI yielded missing data for synovium thickness on MRI. US has advantages over MRI. One of these advantages is its ability of differentiating synovium hypertrophy and hemosiderin deposition, which may not possible with some sequences of MRI in cases of severe deposition of extracellular hemosiderin due to susceptibility artifacts, for example, on gradient-echo images. Although contrast-enhanced MRI could be of value in joints of patients with bleeding disorders with no or minimal hemosiderin deposition it is of reduced value in joints with substantial hemosiderin deposition because the synovium is obscured by dark signal from hemosiderin on post-contrast MRI sequences [31, 32].

Also, the smaller field-of-view of US in comparison to MRI allowed for more detailed evaluation of synovium by US than by MRI. As a result, the assessment of associations of synovium thickness between MRI and gray-scale US was not robust. Of interest, using dynamic contrastenhanced MRI in their study Acharya et al. [9] reported strong correlations between measurements of synovial thickness $(r=0.70, p<0.0001)$ and synovial vascularity $(r=0.73, p<0.0001)$ on power Doppler US and those of MRI. They found a sensitivity of $100 \%$ and a specificity of $94 \%$ for a cutoff of power Doppler US intensity of 1.3 decibels in 17 joints of children and adults with hemophilia $\mathrm{A} / \mathrm{B}$ and other inherited bleeding disorders with/ without a history of hemarthrosis and ages ranging from 6 to 60 years.

A limitation of the study was the unavailability of accurate information about timing of the bleeding history given the retrospective nature of this study. Synovial vascularity scores used in this study were based on the assessment of reactive synovitis which would be indirectly related to recent bleeding compared with previous bleeding history. Previous studies have addressed the lack of accuracy of patients' reports on occurrence of joint bleeds [33], which favours the use of the Hemophilia Joint Health Score (HJHS) as a more objective tool for assessment of the joint health of patients with hemophilia. The HJHS scores was the one of the diagnostic outcome measures used in our study for this reason.

The remaining correlations between subjective assessment of synovitis (scoring) and clinical (HJHS total score and score of items) and radiological (Pettersson X-ray) scores were all poor. These results raise the importance of complementary utilization of physical examination and US as part of clinical practice as previously reported [34]. Note should be made that the synovial hypertrophy scores used in this study were based on the location with maximal synovial thickness in the joint, whereas many of the HJHS items assessed represented either different items (e.g. swelling on HJHS vs joint effusion/hemarthrosis \pm synovial hypertrophy on gray-scale US) or conjoint soft tissue and osteochondral joint findings (e.g. extension and flexion loss, pain vs multiple items on grayscale US). Furthermore, synovial vascularity scores used in this study were based on the assessment of reactive 
synovitis which would be indirectly related to synovial hypertrophy.

The lack of a reference standard for measurements of synovial vascularity for which contrast would have to have been administered to patients is a limitation of this study. For this reason, in addition to superposition of hemosiderin deposition to synovium in many examinations, it was difficult to measure the synovial thickening on corresponding MRI examinations. Nevertheless, the use of contrast-enhanced MRI for assessment of joints of patients with hemophilia is not the standard practice in many centers [31] and was not part of this study. Second, the imaging data from the cohorts in Canada and China were obtained in different timeframes which could have posed differences in quality of imaging between the Canadian and Chinese centers. Nevertheless, the types of probes, US scanner manufacturer, protocol and technical parameters used were similar which made comparable the datasets Third, the HJHS scores used in the China cohorts (sites 1 and 2) and Canada (site 3) cohorts were slightly different (versions 2.1 and 2.0, respectively). Also, elbow joints were only assessed in the China cohorts. Finally, spectrum bias was noted in the cohorts considering that overall the disease burden was significantly greater in the China cases and the joints studied in the Canadian cases were predominantly ankles.

The excellent inter-reader reliability noted for the use of different color Doppler methods (subjective vs objective) for assessment of the maximum degree of synovial vascularity (Table 3) supports the utilization of synovitis scores for assessment of blood-induced arthropathies in clinical practice.

Our study shows moderate to substantial associations between quantitative degree of synovial vascularity on color Doppler and extent of synovial hypertrophy on gray-scale US on data from both Canada and China, but most notably for China data (Table 4). These results raise the possibility that reactive synovitis can help in determining timing of prior joint bleeds (clinical/subclinical) if color Doppler measurements are obtained close in time to the date of the joint bleed. This hypothesis should be investigated in prospective studies with objective criteria being defined upfront.

The overall non-significant correlations observed between measurements of degree of synovial vascularity by color Doppler US and HJHS scores (Table 6) point out to the need for complementary use of US and physical examination in clinical practice. This is due to the fact that different domains (individual vs conjoint joint findings) require evaluation by different diagnostic tests.

Although demographic results of this study have shown that overall the average gray-scale US thickness of single-layer synovium was smaller for Canada data than for China data, results of Table 4 could well represent differences in the available data in the Canadian and Chinese cohorts. In the Canadian cohort, 8 knees and 21 ankles were examined whereas in the Chinese cohort, 11 knees, 12 ankles and 11 elbows were examined with minimal to mild disease noted in the Canadian cohort in contrast to the degree of severity of arthropathy in the Chinese cohort) which resulted in statistically significant $r$ value levels that most likely are not clinically meaningful $(r<0.50)$.

\section{Conclusion}

Color Doppler US is a valuable scoring method for evaluating reactive synovitis in joints of subjects with inherited bleeding disorders and holds potential for assessing post-bleed reactive synovitis in a generalizable worldwide way once further information on its association with timing of the joint bleed becomes available in the literature.

\section{Abbreviations}

ANOVA: Analysis of Variance; HJHS: Hemophilia Joint Health Score; ICCs: Intraclass Correlation Coefficients; JIA: Juvenile Idiopathic Arthritis; MHz: MegaHertz; MRI: Magnetic Resonance Imaging; PACS: Picture Archive Communications Systems; ROIs: Regions of Interest; SD: Standard Deviation; US: Ultrasound; VWD: Von Willebrand disease.

\section{Supplementary Information}

The online version contains supplementary material available at https://doi. org/10.1186/s13244-021-01043-0.

Additional file 1. Supplementary Tables. Table 1. MRI protocols used in the three participating centers. Table 2. Demographic and clinical characteristics of study subjects.

\section{Acknowledgements}

We thank the Canadian Institutes of Health Research (CIHR Planning Grant, Musculoskeletal Health, Arthritis, Skin and Oral Health, 201302PMH, application \#303291, title: "Developing Interdisciplinary Knowledge Exchange Capacity between Canada and China on Imaging Outcomes for Hemophilic Joints") and Baxalta-Shire (Investigator-Initiated Grant, title: "Educational-Research Planning Meeting on Hemophilic Arthropathy between SickKids, Toronto, Canada and Beijing Children's Hospital, China") for the financial support for the China component of the study. We thank Bayer Healthcare Inc (InvestigatorInitiated Grant, title: "Measurement Properties of Ultrasound for Assessment of Hemophilic Arthropathy. MRI Correlation") for the financial support for the Canadian component of the study.

\section{Authors' contributions}

ASD, NZ, AFZ, AM conceived and designed the study. NZ, SY, AFZ collected and archived most of the images used in the study. NZ, SY, AFZ, AM, AZ, JJ, $A H, Y L, F Z, P H, S S, R M$ acquired, analyzed and interpreted the data. NZ, SY, AFZ, AM, AZ, JJ, AH, YL, FZ, VB, PH, SS, BL, VB, RW, JS, YP, RM, ASD contributed to the observation and discussion of the results shown in this work, critically reviewed the manuscript for important intellectual content and approved the final version for publication. YP is responsible for the cases management and imaging data collection of site 1. NZ and ASD wrote up the manuscript draft. ASD supervised AFZ during the conduct of the study. All authors read and approved the final manuscript. 


\section{Funding}

The Canadian Institutes of Health Research and Baxalta-Shire provided financial support for the China component of the study. Bayer Healthcare Inc provided financial support for the Canadian component of the study.

\section{Availability of data and materials}

All data generated or analyzed during this study are included in this published article and its supplementary information files.

\section{Declarations}

\section{Ethics approval and consent to participate}

This study was approved by the Research Ethics Board committees of the participating Institutions (The Hospital for Sick Children, Beijing Children's Hospital and Nanfang Hospital). Informed consent or assent was obtained from all parents and/or patients who participated in the original prospective studies conducted in Canada and China.

\section{Consent for publication}

Available upon request.

\section{Competing interests}

ASD: Research support by Bayer Healthcare Inc (Research Grant), Novo Nordisk Healthcare Inc (Research Grant), Terry Fox Foundation (Research Grant), Physician's Services Incorporation (Research Grant). Advisory Committee: International Prophylaxis Study Group (not for profit). OMERACT SIG in MRI in JIA (not for profit), Canadian Committee of Point-of-Care Ultrasound (not for profit). Consultant: Baxter Healthcare Inc, Pfizer Healthcare Inc. PH: Patent on HJHS v2.1 (Hemophilia Joint Health Score) used under license by the Hospital for Sick Children. VB: Chair of the International Prophylaxis Study Group (IPSG), a co-operative study group that is funded by Education grants from Bayer Healthcare, Bioverativ/Sanofi, Novo Nordisk, Pfizer, Shire/Takeda and Spark Therapeutics to the Hospital for Sick Children ("SickKids") Foundation. Received personal fees from Amgen, Bayer, Novo Nordisk, Pfizer, Roche, Shire/Takeda, Octapharma, and Research support Grants from Novo Nordisk, Bioverativ/ Sanofi and Shire/Takeda. RW: Receives research support from Bayer-China. BL: Received honoraria from Takeda China and Bayer China. NZ, AFZ, AM, SY, AZ, $J$ J, AH, YL, FZ, SS, JS, VB, YP and RM: no competing interests.

\section{Author details}

${ }^{1}$ Department of Radiology, Beijing Children's Hospital, Capital Medical University, National Center for Children's Health, Beijing, China. ${ }^{2}$ Department of Ultrasound, Chengdu Women's and Children's Central Hospital, Sichuan, China. ${ }^{3}$ Department of Pediatric Haematology, Amsterdam UMC, University of Amsterdam, Amsterdam, Netherlands. ${ }^{4}$ Department of Radiology, Nanfang Hospital, Guangzhou, China. ${ }^{5}$ Department of Rehabilitation, The Hospital for Sick Children, Toronto, ON, Canada. ${ }^{6}$ Service of Physiotherapy, St Paul's Hospital, Providence Health Care, Vancouver, BC, Canada. ${ }^{7}$ Department of Paediatrics, University of Toronto, Toronto, ON, Canada. ${ }^{8}$ Division of Haematology/Oncology, The Hospital for Sick Children, Toronto, ON, Canada. ${ }^{9}$ Department of Diagnostic Imaging, Research Institute, The Hospital for Sick Children, University of Toronto, 555 University Ave, Toronto, ON M5G 1X8, Canada. ${ }^{10}$ Haematology/Oncology Center, Beijing Children's Hospital, Capital Medical University, National Center for Children's Health, Beijing, China. ${ }^{11}$ Division of Haematology and Transfusion Medicine, Children's Hospital of Eastern Ontario, Ottawa, ON, Canada. ${ }^{12}$ Division of Family and Community Medicine, University of Toronto, Toronto, ON, Canada. ${ }^{13}$ Department of Medical Imaging, University of Toronto, Toronto, ON, Canada.

Received: 16 November 2020 Accepted: 21 June 2021 Published online: 25 September 2021

\section{References}

1. Srivastava A, Brewer A, Mauser-Bunschoten E et al (2013) Guidelines for the management of hemophilia. Hemophilia 19(1):e1-47

2. Melchiorre D, Manetti M, Matucci-Cerinic M (2017) Pathophysiology of hemophilic arthropathy. J Clin Med 6:63
3. Pulles AE, Mastbergen SC, Schutgens RE, Lafeber FP, van Vulpen LF (2017) Pathophysiology of hemophilic arthropathy and potential targets for therapy. Pharmacol Res 115:192-199

4. Kraft J, Blanchette V, Babyn P et al (2012) Magnetic resonance imaging and joint outcomes in boys with severe hemophilia A treated with tailored primary prophylaxis in Canada. J Thromb Haemost 10(12):2494-2502

5. Doria AS (2010) State-of-the-art imaging techniques for the evaluation of haemophilic arthropathy: Present and future. Haemophilia 16(Suppl. 5):107-114

6. Adler RS, Bell DS, Bamber JC, Moskovic E, Thomas JM (1999) Evaluation of soft-tissue masses using segmented color Doppler velocity images: preliminary observations. AJR Am J Roentgenol 172:781-788

7. Newman JS, Liang TJ, McCarthy CJ, Adler RS (1996) Power Doppler sonography of synovitis: assessment of therapeutic responsepreliminary bservations. Radiology 198:582-584

8. Acharya SS (2008) Hemophilic joint disease-current perspective and potential future strategies. Transfus Apher Sci 38(1):49-55

9. Acharya SS, Schloss R, Dyke JP et al (2008) Power Doppler sonography in the diagnosis of hemophilic synovitis-a promising tool. J Thromb Haemost 6(12):2055-2061

10. Bhat $\mathrm{V}$, Olmer $\mathrm{M}$, Joshi $\mathrm{S}$ et al (2015) Vascular remodeling underlies rebleeding in hemophilic arthropathy. Am J Hematol 90(11):1027-1035

11. Cooke EJ, Zhou JY, Wyseure T et al (2018) Vascular permeability coincide with inflammatory and reparative processes after joint bleeding in factor VII-deficient mice. Thromb Haemost 118(6):1036-1047

12. Zhou JY, Barnes RFW, Foster G, lorio A, Cramer TJ, von Drygalski A (2019) Joint bleeding tendencies in adult patients with hemophilia: it's not all pharmacokinetics. Clin Appl Thromb Hemost 25:1076029619862052

13. Rubin JM, Bude RO, Carson PL, Bree RL, Adler RS (1994) Power Doppler US: a potentially useful alternative to mean frequency-based color Doppler US. Radiology 190(3):853-856

14. Terslev L, Torp-Pedersen S, Qvistgaard E, Danneskiold-Samsoe B, Bliddal $H$ (2003) Estimation of inflammation by Doppler ultrasound: quantitative changes after intra-articular treatment in rheumatoid arthritis. Ann Rheum Dis 62(11):1049-1053

15. Torp-Pedersen S, Christensen R, Szkudlarek M et al (2015) Power and color Doppler ultrasound settings for inflammatory flow: impact on scoring of disease activity in patients with rheumatoid arthritis. Arthritis Rheumatol 67(2):386-395

16. Roosendaal G, Van Rinsum AC, Vianen ME, Van den Berg HM, Lafeber FPJG, Bijlsma JWJ (1999) Haemophilic arthropathy resembles degenerative rather than inflammatory joint disease. Histopathology 34(2):144-153

17. Jaganathan S, Gamanagatti S, Goyal A (2011) Musculoskeletal manifestations of hemophilia: imaging features. Curr Probl Diagn Radiol 40:191-197

18. Doria AS, Kiss MH, Lotito AP et al (2001) Juvenile rheumatoid arthritis of the knee: evaluation with contrast-enhanced color Doppler ultrasound. Pediatr Radiol 31(7):524-531

19. Naredo E, Collado P, Cruz A et al (2007) Longitudinal power Doppler ultrasonographic assessment of joint inflammatory activity in early rheumatoid arthritis: predictive value in disease activity and radiologic progression. Arthritis Care Res 57(1):116-124

20. Van Meegeren ME, Roosendaal G, Jansen NW, Lafeber FP, Mastbergen SC (2013) Blood-induced joint damage: the devastating effects of acute joint bleeds vs micro-bleeds. Cartilage 4(4):313-320

21. Valentino LA (2010) Blood-induced joint disease: the pathophysiology of hemophilic arthropathy. J Thromb Haemost 8(9):1895-1902

22. Van Vulpen LFD, Holstein K, Martinoli C (2018) Joint disease in haemophilia: Pathophysiology, pain and imaging. Haemophilia 24(Suppl 6):44-49

23. Acharya SS, Kaplan RN, Macdonald D, Fabiyi OT, DiMichele D, Lyden D (2011) Neoangiogenesis contributes to the development of hemophilic synovitis. Blood 117(8):2484-2493

24. Backhaus M, Ohrndorf S, Kellner H et al (2009) Evaluation of a novel 7-joint ultrasound score in daily rheumatologic practice: a pilot project. Arthritis Rheum 61(9):1194-1201

25. Keshava S, Gibikote S, Mohanta A, Doria AS (2009) Refinement of a sonographic protocol for assessment of haemophilic arthropathy. Haemophilia 15(5):1168-1171 
26. Xavier F, Zhang N, Mohanta A et al (2012) Sonography for assessment of elbows in haemophilic children: a systematic protocol. Rheumatol Curr Res S2:1-9

27. Doria AS, Keshava SN, Mohanta A et al (2015) Diagnostic accuracy of ultrasound for assessment of hemophilic arthropathy: MRI correlation. AJR Am J Roentgenol 204(3):W336-347

28. Feldman B, Funk S, Bergstrom B et al (2011) Validation of a new pediatric joint scoring system from the international hemophilia prophylaxis study group: validity of the hemophilia joint health score (HJHS). Arthritis Care Res (Hoboken) 63:223-230

29. Altman D (1991) Practical statistics for medical research. Chapman and Hall, London

30. Tolend M, Kellenberger C, Junhasavasdikul T, et al (2018) Inter-reader reliability study of a new MRI scoring system for TMJ in JIA from the MRI in JIA according to the use of measurement aids: Special Interest Group in OMERACT [Abstract]. In: Proceedings of society of pediatric radiology annual meeting, Nashville, TN
31. Lundin B, Berntorp E, Pettersson H et al (2007) Gadolinium contrast agent is of limited value for magnetic resonance imaging assessment of synovial hypertrophy in hemophiliacs. Acta Radiol 48(5):520-530

32. Nägele M, Kunze V, Hamann M et al (1994) Gd-DTPA-verstärkte MRT; klinische und röntgenologische Korrelation [Hemophiliac arthropathy of the knee joint. Gd-DTPA-enhanced MRl; clinical and roentgenological correlation]. Rofo 160(2):154-158

33. Simpson ML, Valentino LA (2012) Management of joint bleeding in hemophilia. Expert Rev Hematol 5(4):459-468

34. Poonnoose PM, Hilliard P, Doria AS et al (2016) Correlating clinical and radiological assessment of joints in haemophilia: results of a cross sectional study. Haemophilia 22(6):925-933

\section{Publisher's Note}

Springer Nature remains neutral with regard to jurisdictional claims in published maps and institutional affiliations.

\section{Submit your manuscript to a SpringerOpen ${ }^{\circ}$ journal and benefit from:}

- Convenient online submission

- Rigorous peer review

- Open access: articles freely available online

- High visibility within the field

- Retaining the copyright to your article

Submit your next manuscript at $\boldsymbol{\nabla}$ springeropen.com 